\title{
Complications and outcome in patients with aneurysmal subarachnoid haemorrhage: a prospective hospital based cohort study in The Netherlands
} Y B W E M Roos, R J de Haan, L F M Beenen, R J M Groen, K W Albrecht,
M Vermeulen
Department of

Neurology, Academic Medical Centre,

University of

Amsterdam,

Amsterdam, The

Netherlands

Y B W E M Roos

$M$ Vermeulen

Department of

Neurosurgery

K W Albrecht

Department of Clinical

Epidemiology and

Biostatistics

R J de Haan

Department of

Neurosurgery,

Academic Hospital of

the Free University,

Amsterdam, The

Netherlands

L F M Beenen

Department of

Neurosurgery,

Slotervaart General

Hospital, Amsterdam,

The Netherlands

R J M Groen

Correspondence to:

Dr Yvo Roos, Department of

Neurology, Academic

Medical Centre,

Meibergdreef 9, $1105 \mathrm{AZ}$

Amsterdam-zuidoost, The

Netherlands

email

Y.B.Roos@AMC.UVA.NL

Received 23 April 1999 and in revised form

9 September 1999

Accepted 12 October 1999

\begin{abstract}
Objective-The aim of this study was to investigate prospectively in an unselected series of patients with an aneurysmal subarachnoid haemorrhage what at present the complications are, what the outcome is, how many of these patients have "modern treatment"- that is, early obliteration of the aneurysm and treatment with calcium antagonists-what factors cause a delay in surgical or endovascular treatment, and what the estimated effect on outcome will be of improved treatment.
\end{abstract}

Methods-A prospective, observational cohort study of all patients with aneurysmal subarachnoid haemorrhage in the hospitals of a specified region in The Netherlands. The condition on admission, diagnostic procedures, and treatments were recorded. If a patient had a clinical deterioration, the change in Glasgow coma score (GCS), the presence of focal neurological signs, the results of additional investigations, and the final diagnosed cause of the deterioration were recorded.

Clinical outcome was assessed with the Glasgow outcome scale (GOS) at 3 month follow up. In patients with poor outcome at follow up, the cause was diagnosed.

Results-Of the 110 patients, 47 (43\%) had a poor outcome. Cerebral ischaemia, 31 patients $(28 \%)$, was the most often occurring complication. Major causes of poor outcome were the effects of the initial haemorrhage and rebleeding in $34 \%$ and $30 \%$ of the patients with poor outcome respectively. Of all patients $102(93 \%)$ were treated with calcium antagonists and 45 (41\%) patients had early treatment to obliterate the aneurysm. The major causes of delay of treatment were a poor condition on admission or deterioration shortly after admission, in $31 \%$ and $23 \%$ respectively.

Conclusions-In two thirds of the patients with poor outcome the causes of poor outcome are the effects of the initial bleeding and rebleeding. Improved treatment of delayed or postoperative ischaemia will have only minor effects on the outcome of patients with subarachnoid haemorrhage. (F Neurol Neurosurg Psychiatry 2000;68:337-341)
Keywords: aneurysmal subarachnoid haemorrhage; timing of surgery; complications

Reviews on aneurysmal subarachnoid haemorrhage suggest that with modern treatment strategies, cerebral ischaemia is the only complication that has remained as a cause of poor outcome. ${ }^{1}$ The modern treatment includes treatment with calcium antagonists, such as nimodipine, to prevent cerebral ischaemia, and by far the most important, the prevention of rebleeding by occlusion of the aneurysm within 3 days after the initial bleeding. $^{2-4}$

In a previous study, however, we found that in neurosurgical centres using modern treatment strategies, despite the aim to prevent rebleeding by early surgery, $45 \%$ of the patients was not operated on within 3 days after the initial aneurysmal subarachnoid haemorrhage and that rebleeding was still the major cause of poor outcome. ${ }^{5}$ Similar results were found in two recently published population based studies in King County, USA and in greater Cincinnati, USA. ${ }^{6}$ None of these studies, however, investigated which factors caused the delay in surgery.

The aim of the present study was therefore to reconfirm in an unselected regional cohort of patients with aneurysmal subarachnoid haemorrhage our previous results concerning the timing of surgery and to investigate prospectively which factors are actually responsible for the delay in surgery. Additionally, we investigated how many patients are treated nowadays with calcium antagonists to prevent cerebral ischaemia, what the complications after aneurysmal subarachnoid haemorrhage are at present, and what the outcome of patients is in such a series. Finally, we estimated what the effect might be of improved treatment of complications after subarachnoid haemorrhage on the final outcome.

\section{Patients and methods}

STUDY DESIGN

The study design was a prospective cohort study of consecutively admitted patients with aneurysmal subarachnoid haemorrhage in all hospitals in two regions of the Netherlands, NoordHolland and Flevoland (appendix). According to official figures from the Dutch Central Bureau of Statistics (CBS), a governmental institution, this region has about 2000000 
inhabitants. All 12 regional hospitals in this region refer their patients with aneurysmal subarachnoid haemorrhage for neurosurgical interventions to the three neurosurgical units in Amsterdam. These neurosurgical units are all situated less than $85 \mathrm{~km}$ (50 miles) from the most remote regional hospital. All three neurosurgical units adhere to the same management protocol which includes treatment with the calcium antagonist nimodipine $(2 \mathrm{mg} /$ hour intravenously or $6 \times 60 \mathrm{mg} /$ day orally), hypervolaemic, hypertensive treatment to prevent delayed cerebral ischaemia (minimum of 31 fluid intake daily), and early surgery to prevent rebleeding. ${ }^{49}$ The three neurosurgical units in Amsterdam are all teaching hospitals and within each centre at least three neurosurgeons operate on cerebral aneurysms.

PATIENT SELECTION

All patients with the diagnosis of aneurysmal subarachnoid haemorrhage, primarily admitted in the participating centres, were prospectively studied during a 1 year period (February 1996-February 1997). The diagnosis was based on clinical signs and symptoms and an aneurysmal bleeding pattern on the initial $\mathrm{CT} .{ }^{10}$ If the initial CT was negative and subsequent CSF examination positive (xanthochromia confirmed by spectrophotometry) an aneurysm had to be confirmed by angiography before the patient could be included. ${ }^{11}$ Patients with a perimesencephalic bleeding pattern or another non-aneurysmal pattern of haemorrhage on CT were excluded. ${ }^{12}$

DATA COLLECTION AND MANAGEMENT

Data registration was done in the participating centres by two research nurses on two portable personal computers equipped with an electronic case record form/database program specially developed for this study. This program generated electronic forms in which all patients' data were directly recorded.

Registration of baseline and clinical data started on admission in the hospital where the patient was seen initially. The following baseline characteristics were recorded: age; sex; date of aneurysmal subarachnoid haemorrhage, and date of admission. Clinical details recorded at baseline were the Glasgow coma scale (GCS) and the grade of subarachnoid haemorrhage (World Federation of Neurological Surgeons (WFNS)) score on admission ${ }^{13}{ }^{14}$; the results of CT, CSF examination, and the location(s) of (an) aneurysm(s) on angiography.

After admission patients were followed up on a day to day basis during their whole period in hospital. All details on treatments used and management strategies (such as fluid management, drug therapies, operative interventions, consulted specialists, and transfers to other hospitals or other wards such as intensive care) and all additional diagnostic procedures (like blood samples, radiological investigations) were recorded.

Every time a patient had a clinical deterioration an additional form was filled out: the date of the deterioration, the change in GCS, the onset of the deterioration (sudden versus gradually), the presence of focal neurological signs, and the final diagnosed cause of the deterioration after appropriate investigations were recorded. The final assessment of patient's complication was performed by two of us (YR and MV), using clinical data, CT data, and necropsy results. Rebleeding and delayed cerebral ischaemia were defined as previously described as definite rebleeding or probable rebleeding and definite infarction or probable infarction. ${ }^{15}$ Hydrocephalus was defined as the gradual deterioration of consciousness with CT evidence of hydrocephalus and no other explanation for deterioration. ${ }^{15}$ Postoperative ischaemia was defined as a change in the level of consciousness or the development of focal neurological signs noticed immediately after recovery from anaesthesia compared with the preoperative status, with no evidence of rebleeding or hydrocephalus on CT or at necropsy

Functional health outcome was assessed with the Glasgow outcome scale (GOS).$^{16}$ The GOS is an index score ranging from 1 (death) to 5 (good recovery). Death, persistent vegetative state, and severe disability on the GOS were combined as poor outcome and moderate disability and good recovery were recorded on the study forms as good outcome. In patients with poor outcome at 3 month follow up, one of us (YR) reviewed the medical record of the patient to determine and categorise the actual cause for this poor outcome in five major categories: initial bleed; rebleeding; cerebral ischaemia; operative complications or other (non-neurological) complications, which had to be specified. Poor outcome caused by the effect of the initial bleeding was defined as impaired consciousness or focal neurological signs existing since the initial bleed, without signs of rebleeding, ischaemia, or hydrocephalus on CT or at necropsy.

\section{ETHICAL CONSIDERATIONS}

The study protocol was approved by the local legal advisor or ethics committee of each participating centre. Informed consent was asked for in all patients (or legal representative) with the exception of those who died shortly after admission, in which case there was no need for active participation of the patients or their relatives.

During the whole study period patients were identified by a unique patient identification number (PIN) to protect privacy. The code key to this PIN number remained the responsibility of the treating physician of the hospital where the patient was first seen during the study period and was destroyed after completion of the whole data set.

\section{Results}

During the 1 year study period 110 patients with an aneurysmal subarachnoid haemorrhage were admitted to the participating regional hospitals and neurosurgical centres. Table 1 shows the baseline characteristics of these patients, which are comparable with baseline characteristics in other population 
Table 1 Baseline characteristics ( $n=110$ patients)

\begin{tabular}{lc}
\hline Age (mean y, range) & $56(29-89)$ \\
Males (n) & 39 \\
Females (n) & 71 \\
Glasgow coma score on admission & \\
$\quad$ (median, range) & $14(3-15)$ \\
WFNS (\%): & 46 \\
$\quad$ Grade 1 & 24 \\
Grade 2 & 0 \\
Grade 3 & 21 \\
Grade 4 & 9 \\
Grade 5 & \\
Time lapse between SAH and admission & 0 \\
$\quad$ (median days) & \\
\hline
\end{tabular}

$\mathrm{SAH}=$ subarachnoid haemorrhage.

Table 2 Complications after admission

\begin{tabular}{llll}
\hline & & & $\begin{array}{l}\% \text { of patients with } \\
\text { poor outcome caused } \\
\text { by this complication }\end{array}$ \\
\hline Rebleeding & 18 & 16 & 78 \\
Delayed cerebral ischaemia & 19 & 17 & 42 \\
Post operative ischaemia & 12 & 11 & 25 \\
Other & 18 & 16 & 34 \\
\hline
\end{tabular}

studies in aneurysmal subarachnoid haemorrhage. Because the study region covered by the participating hospitals has about 2000000 inhabitants, this hospital based study shows a yearly aneurysmal subarachnoid haemorrhage incidence of 5.5 patients/100 000 inhabitants.

Table 2 shows the occurrence of complications after admission. There were more patients with cerebral ischaemia than with rebleeds but rebleeding had more impact on outcome. In $78 \%$ of the patients who had a rebleed this complication was the cause of poor outcome, whereas other complications caused a poor outcome less often. Of the complications in the "other" group, hydrocephalus was that most often occurring.

In the group of 18 patients with a rebleed, seven had this complication within 2 days after the initial haemorrhage, four on the day of the haemorrhage. Five rebleeds occurred in patients in whom treatment of the aneurysm had been postponed. In three patients with a rebleed, the initial bleed had not been recognised as an aneurysmal subarachnoid haemorrhage. Two patients had a rebleed during surgery and one after surgery. One patient had a rebleed after a negative angiography. Ten of the 18 patients who had a rebleeding were in a good clinical condition (WFNS 1 or 2) when surgery was considered. In this group of "good grade" patients, four patients had the rebleed on the day of the initial bleeding, two on the next day, and one during surgery. Three patients had a delay in surgery because the initial aneurysmal subarachnoid haemorrhage had not been recognised and the last patient had a negative first angiography.

Of all patients 47 (43\%) had poor outcome, as expected outcome was related to the clinical condition on admission; 24 of the 77 "goodgrade" (WFNS 1 or 2) patients (31\%) had a poor outcome. Table 3 shows the causes of poor outcome. In two thirds of the patients with poor outcome, the cause was the effect of the initial haemorrhage or rebleeding.

Table 4 shows the timing of both surgery and coiling to occlude the aneurysm. During the inclusion period (February 1996-February 1997) coiling of aneurysms in the acute phase just started in this region of The Netherlands. Three patients had coils placed within 3 days after their SAH. Another seven patients had coiling of their aneurysm after a delay of at least 3 days. In these patients the aneurysm was firstly considered unsuitable for surgery; thereafter the question was whether coiling was an option. This led to delayed coiling due to delayed consultation. Surgical clipping was the preferred treatment to occlude the aneurysm in 66 patients $(60 \%)$. However, in 24 of these patients (36\% of the patients with surgery) surgery was postponed for more than 3 days after aneurysmal subarachnoid haemorrhage and 34 of all patients $(31 \%)$ had no surgery or coiling of the aneurysm.

In $102(93 \%)$ of the patients treatment with calcium antagonists was started. In seven of the remaining eight patients calcium antagonists were not given because these patients were in a poor clinical condition on admission; all died within 3 days after the initial bleeding.

Table 5 lists the causes of delay of treatment to occlude the aneurysm. Deterioration after admission included rebleeding, ischaemia, and hydrocephalus. Delay in referral to the neurosurgical centre was caused by a delay in recognition of the aneurysmal subarachnoid haemorrhage before admission in five patients and in four patients after admission to the regional hospital. Of the eight patients with a negative angiogram, in two an aneurysm was demonstrated after angiography had been repeated. In 10 patients the aneurysm was considered difficult to operate on and in seven patients coiling was preferred. The planning of this procedure led to delayed treatment.

Table 3 Causes of poor outcome

\begin{tabular}{lcr}
\hline & $n=47$ & $\%$ \\
\hline Initial bleed & 16 & 34 \\
Rebleeding & 14 & 30 \\
Delayed cerebral ischaemia & 8 & 17 \\
Post operative ischaemia & 3 & 6 \\
Other & 6 & 13 \\
\hline
\end{tabular}

Table 4 Treatment to occlude the aneurysm

\begin{tabular}{lll}
\hline & $\begin{array}{l}\text { No of } \\
\text { patients }\end{array}$ & $\begin{array}{l}\% \text { of total } \\
n=110\end{array}$ \\
\hline Surgery & 66 & 60 \\
Within 3 days & 42 & \\
After 3 days & 24 & \\
Coiling & 10 & \\
Within 3 days & 3 & \\
After 3 days & 7 & 31 \\
No surgery or coiling & 34 & \\
\hline
\end{tabular}

Table 5 Causes of delayed treatment to occlude the aneurysm

\begin{tabular}{lrr}
\hline & \multicolumn{1}{c}{$n$} & $\%$ \\
\hline Poor condition on admission & 20 & 31 \\
Deterioration after admission & 15 & 23 \\
Delay in referral & 9 & 14 \\
Negative angiography & 8 & 12 \\
Complex aneurysm & 10 & 15 \\
Logistic & 3 & 5 \\
Total & 65 & 100 \\
\hline
\end{tabular}




\section{Discussion}

The incidence of aneurysmal subarachnoid haemorrhage found in this study, 5.5/100 000 a year, is not much different from estimates of this incidence in the Dutch population. Apparently, few patients die before reaching hospital and in a few patients aneurysmal subarachnoid haemorrhage is not recognised. Similarly, in a population based study from King County, Washington, USA only $3 \%$ of patients with aneurysmal subarachnoid haemorrhage had died before admission. ${ }^{6}$ Therefore, the results of this study are probably representative for all patients with aneurysmal subarachnoid haemorrhage.

The most often occurring complication is cerebral ischaemia, but the proportion of patients with a rebleed is still $16 \%$. However, cerebral ischaemia is not the major cause of poor outcome as the effects of the initial bleed and rebleeding account for two thirds of all poor outcomes.

This study shows that despite aiming at modern treatment strategies the proportion of patients with poor outcome is still $43 \%$. This percentage is much higher than in other studies but these studies have usually been carried out in neurosurgical centres and often consist of randomised clinical trials. Therefore, these figures come from highly selected groups of patients.

Modern treatment consists of early obliteration of the aneurysm and administration of calcium antagonists. Calcium antagonists were given to most of the patients (93\%) but early treatment of the aneurysm was carried out in less than half.

We expected that logistic reasons such as, for instance, a delay in angiography at the department of radiology or difficulties in finding an available theatre or a surgeon on short notice would be the major causes of delay. Logistic reasons however, seemed to play hardly any part. The major causes of delaying treatment were the condition of the patient on admission and deterioration from complications such as rebleeding and ischaemia occurring before the planned surgery or coiling to occlude the aneurysm.

The proportion of patients with a rebleed is still high but not all these rebleeds can be prevented by early treatment of the aneurysm. In the group of 18 patients with a rebleeding, in seven this occurred within 2 days of the initial haemorrhage and in two during surgery. Very rigorous treatment to occlude the aneurysm as early as possible in all patients, irrespective of the clinical condition on admission, may half the number of patients with rebleeding. As rebleeding is the cause of poor outcome in about $30 \%$ of the patients, this management would lead to a reduction of poor outcome in about $15 \%$.

Similarly, if it is possible to reduce the occurrence of cerebral ischaemia by $50 \%$ by a new treatment, irrespective of whether ischae- mia developed unrelated to surgery or during or immediately after surgery, the reduction of poor outcome will probably be less than $15 \%$. However, these reductions are difficult to achieve. This study shows that treatment to prevent rebleeding is usually postponed because of the poor clinical condition of the patient on admission or because of deterioration shortly after admission. The results of surgery in this group of patients are less good than in patients who undergo surgery in a better condition. Moreover, in this group of patients outcome may be poor despite prevention of rebleeding, therefore the impact of surgery on outcome will probably be much less than the above estimated $15 \%$. In this study only three of the 18 rebleeds could have been prevented if surgery had been performed in all good grade patients on the same day that the aneurysm was demonstrated. It might be that better results can be achieved by using endovascular techniques to occlude the aneurysm in the acute phase, but also with this treatment the devastating effects of the initial bleed or early complications will remain.

A new effective treatment for cerebral ischaemia might have a different impact in the different types of cerebral ischaemia, as the mechanism of postoperative ischaemic damage is probably not the same as in delayed cerebral ischaemia. If treatment works only on delayed cerebral ischaemia and is very effective by reducing half of the occurrences of this type of ischaemia, the reduction of poor outcome will not be more than $10 \%$. If the effectiveness is less-for instance a 30\% reduction of cerebral ischaemia, which is still considerable - it will be extremely difficult to demonstrate an effect of this treatment on outcome. To demonstrate a beneficial effect of such a treatment on outcome at least 2825 patients have to be randomised in a controlled trial. An even larger number of patients would be necessary if treatment is effective on postoperative ischaemia only.

The results of this study do not support the suggestion expressed in reviews that with modern treatment, delayed cerebral ischaemia is the only problem that has to be dealt with to improve outcome in patients with aneurysmal subarachnoid haemorrhage. The explanation is that in two thirds of the patients with poor outcome the causes are the effects of the initial bleeding and rebleeding. Only if these effects can be reduced, will the outcome of all patients improve. Further improvement of the treatment of delayed cerebral and postoperative ischaemia will only have minor effects on the outcome of patients with aneurysmal subarachnoid haemorrhage.

We thank M Mechielsen and A Vet for their excellent data management. We also thank the pharmaceutical firm Pharmacia and Upjohn for their financial support of this study. 


\section{Appendix: list of participating centres (in alphabetical order)}

Academic Medical Centre (AMC), Amsterdam (Y Roos, KW Albrecht, M Vermeulen, RJ de Haan)

BovenIJ Ziekenhuis, Amsterdam (A Janse)

Flevoziekenhuis, Almere (AR Kuhler, H Kuiper)

Free University Hospital (VU), Amsterdam (LFM Beenen, JFNM Ploegmakers)

Kennemer Gasthuis, Haarlem (RMCM Janssen, JAM Kuster)

Kennemer Gasthuis, IJmuiden (JA Don, HCW Hoff)

Medisch Centrum Alkmaar (MCA), Alkmaar ( MM Veering)

Onze Lieve Vrouwe Gasthuis (OLVG), Amsterdam (P Verlooy)

Rode Kruis Ziekenhuis, Beverwijk (HJJM vd Werd)

Sint Lucas Andreas Ziekenhuis, Amsterdam (JLM Hellenberg Hubar, JAL Vanneste)

Slotervaart Hospital, Amsterdam (RJM Groen)

St Gemini Ziekenhuis, Den Helder (JG Kok)

St Ziekenhuis De Heel, Zaandam (AJ Prazsky, A Kopenaal)

Waterlandziekenhuis, Purmerend (CP Zwetsloot)

Westfries Gasthuis, Hoorn (FEAM Bussemaker, GJ Lambrechts)

IJsselmeerziekenhuizen, Lelystad (RJJ Tans)

Ziekenhuis Amstelveen, Amstelveen (P Lanting)
1 Kassell NF. The role of vasospasm in overall outcome from aneurysmal subarachnoid hemorrhage. In: Findlay JM, ed. Cerebral vasospasm. Amsterdam: Elsevier, 1993:27-28.

2 Feigin VL, Rinkel GJ, Algra A, et al. Calcium antagonists in patients with aneurysmal subarachnoid hemorrhage: a systematic review. Neurology 1998;50:876-83.

3 Whitfield PC, Moss H, Ohare D, et al. An audit of aneurysmal subarachnoid haemorrhage: earlier resuscitation and mal subarachnoid haemorrhage: earlier resuscitation and surgery reduces inpatient stay and deaths from
7 Neurol Neurosurg Psychiatry 1996;60:301-6.

4 Mayberg MR, Batjer HH, Dacey R, et al. Guidelines for the management of aneurysmal subarachnoid hemorrhage. A management of aneurysmal subarachnoid hemorrhage. A statement for healthcare professionals from a special writing group of the Stroke Council,
Association. Stroke 1994;25:2315-28.

5 Roos YBWEM, Beenen LFM, Groen RJM, et al. Timing of surgery in patients with aneurysmal subarachnoid haemorrhage; rebleeding is still the major cause of poor outcome in neurosurgical units that aim at early surgery. $f$ Neurol Neurosurg Psychiatry 1997;63:490-3.

6 Longstreth WT Jr, Nelson LM, Koepsell TD, et al. Clinical course of spontaneous subarachnoid hemorrhage: a population-based study in King County, Washington. Neurology 1993;43:712-18

7 Broderick JP, Brott TG, Duldner JE, et al. Initial and recurrent bleeding are the major causes of death following subarachnoid hemorrhage. Stroke 1994;25:1342-7.

8 Hasan D, Vermeulen M, Wijdicks EFM, et al. Effect of fluid intake and antihypertensive treatment on cerebral ischemia after subarachnoid hemorrhage. Stroke 1989;20:1511-15.

9 Pickard JD, Murray GD, Illingworth R, et al. Effect of oral nimodipine on cerebral infarction and outcome after subarachnoid haemorrhage: British aneurysm nimodipine trial. BMF 1989;298:636-42.

10 Vermeulen $M$, van Gijn J. The diagnosis of subarachnoid haemorrhage. I Neurol Neurosurg Psychiatry 1990;53:36572.

11 Vermeulen M, Hasan D, Blijenberg BG, et al. Xanthochromia after subarachnoid haemorrhage needs no revisitation. f Neurol Neurosurg Psychiatry 1989;52:826-8.

12 Rinkel GJ, Wijdicks EF, Hasan D, et al. Outcome in patients with subarachnoid haemorrhage and negative angiography according to pattern of haemorrhage on computed tomography. Lancet 1991;338:964-8.

13 Teasdale G, Jennett B. Assessment of coma and impaired consciousness. A practical scale. Lancet 1974;ii:81-4.

14 Teasdale GM, Drake CG, Hunt W, et al. A universal subarachnoid hemorrhage scale: report of a committee of the World Federation of Neurosurgical Societies. 7 Neurol Neurosurg Psychiatry 1988;51:1457.

15 van Gijn J, Bromberg JEC, Lindsay KW, et al. Definition of initial grading, specific events, and overall outcome in patients with aneurysmal subarachnoid hemorrhage: a survey. Stroke 1994;25:1623-7.

16 Jennett B, Bond $M$. Assessment of outcome after severe brain damage. Lancet 1975; i:480-4. 